www.jmscr.igmpublication.org

Index Copernicus Value: 79.54

ISSN (e)-2347-176x ISSN (p) 2455-0450

crossrefDOI: https://dx.doi.org/10.18535/jmscr/v7i3.76

\title{
Analysis of Multiple Primary Malignant Neoplasms: A Report from a Tertiary Cancer Centre in South India
}

\author{
Authors \\ Kannan Jayaraman*, Ramya Rangarajan \\ *Corresponding Author \\ Kannan Jayaraman
}

\begin{abstract}
Aim: Epidemiological studies have reported a $2-17 \%$ incidence of double malignancies. Cancer predisposition syndromes, common environmental exposure, late effects of radiotherapy and chemotherapy can lead to the development of second primary malignancies. The aim of our study is to report the prevalence of multiple pimary malignant neoplasms in our setting and to review the relevant literature.

Materials and Methods: Details of patients presenting with histologically proven synchronous or metachronous double malignancies between January 2016 and May 2017 were collected. Details like age at the time of presentation, gender, location of the first and second primary malignancy, histopathology of both the malignant neoplasms and treatment given for both the malignant neoplasms were recorded.

Results: The incidence of multiple primary malignant neoplasms was $1.25 \% .68 .75 \%$ of patients developed metachronous double primary malignancy and $31.25 \%$ of patients had synchronous double malignancy. The duration between the development of first and second malignancy ranged between 1 to 30 years. $53.12 \%$ of patients who developed second primary malignancy were males, whereas $46.88 \%$ were females. Head and neck cancer was the most common first and second primary malignant neoplasm. The common histopathologies of the double malignancy were squamous cell carcinoma, adenocarcinoma, transitional cell carcinoma and sarcoma.

Conclusion: A regular follow up, a strong clinical suspicion and thorough evaluation could detect most of the metachronous second malignancies at an earlier stage. An individualised treatment approach is warranted for patients with multiple malignancies. The prognosis of these patients depends on the individual tumor biology and the stage of presentation of the individual tumor.
\end{abstract}

\section{Introduction}

Epidemiological studies have reported a $2-17 \%$ incidence of double malignancies ${ }^{(1-5)}$. The phenomenon of multiple primary malignancies was reported as early as 1889 by Bilroth. Multiple primary malignancies may be synchronous or metachronous $^{(6)}$. The new primary which occurs within 6 months from the time of diagnosis of the first malignancy is synchronous multiple primary neoplasm. Metachronous multiple primary neoplasm occurs more than 6 months from the time of diagnosis of the first neoplasm.

The criteria for diagnosis of double primary malignant neoplasms were put forth by Warren and Gates $^{(7)}$. Histological confirmation of malignancy in both the primary neoplasms is essential. There should be atleast $2 \mathrm{~cm}$ normal mucosa between the two malignancies and the 
probability of one being the metastases of the other should be ruled out. There has been an increase in the burden of second primary malignant neoplasms due to improved diagnostic tests, better screening and surveillance techniques and more sophisticated treatments.

Cancer predisposition syndromes, common environmental exposure, late effects of radiotherapy and chemotherapy can lead to the development of second primary malignancies.

\section{Aim}

The aim of our study is to report the prevalence of multiple pimary malignant neoplasms in our setting and to review the relevant literature.

\section{Materials and Methods}

We analysed the case records of patients who attended the department of oncology between January 2016 and May 2017. Details of patients presenting with histologically proven synchronous or metachronous double malignancies were collected. Patients with two or more malignant neoplasms in different locations which are histopathologically proven were included in the study. Patients without a clear histopathological confirmation of the second tumor were excluded. Details like age at the time of presentation, gender, location of the first and second primary malignancy, histopathology of both the malignant neoplasms and treatment given for both the malignant neoplasms were recorded.

\section{Results}

Out of 2560 patients registered in the department of oncology between January 2016 and May 2017, $32(1.25 \%)$ cases of multiple primary malignant neoplasms were recorded. $31.25 \%$ (10)of total patients had synchronous double malignancy and $68.75 \%$ (21) of patients had metachronous double malignancy (Fig1). The duration between development of first primary malignancy and second primary malignancy ranged between 1 to 30 years with an average of 4.9 years.
The median age of incidence of first primary malignancy was 56 years (range: 33 to 76years). Out of the 32 patients diagnosed with multiple primary malignancies, $53.12 \%$ were males and $46.88 \%$ were females (Fig 2). The most common first primary malignancy was head and neck cancer $(53.13 \%)$ followed by breast cancer (12.5\%) (Fig 3). Other first primary malignancies were cervical cancer $(6.25 \%)$ renal cell carcinoma $(6.25 \%)$,colorectal cancer $(6.25 \%)$, secondaries neck with unknown primary $(3.13 \%)$, prostate cancer $(3.13 \%)$, bladder cancer $(3.13 \%)$, soft tissue sarcoma $(3.13 \%)$ and lung cancer $(3.13 \%)$. The most common histopathology of the first primary malignant neoplasm was squamous cell carcinoma (56.25\%) (Fig 4) followed by adenocarcinoma $(37.5 \%)$, transitional cell carcinoma and sarcoma (3.13\% each). Majority of patients with first primary malignancy were treated with chemoradiation (34.38\%) (Fig 5). All the three major modalities namely surgery, chemotherapy and radiotherapy were used in $21.88 \%$ of patients. $18.75 \%$ of patients were treated with surgery alone. Radiotherapy alone was used in $15.63 \%$ of patients.

The most common second primary malignancy was also head and neck cancer (59.38\%), followed by cervical cancer $(12.5 \%)$, bladder $(6.25 \%)$, endometrium $(6.25 \%)$, ovary $(6.25 \%)$, esophagus $(6.25 \%)$ and breast cancer (3.13\%) (Fig 6). 75\% of second primary malignancies were squamous cell carcinoma followed by adeno carcinoma $(18.75 \%)$ and transitional cell carcinoma (6.25\%). (Fig 7) Chemoradiation was the most common treatment strategy adopted for the second primary (Fig 8). 


\section{JMSCR Vol||07||Issue||03||Page 414-419||March}

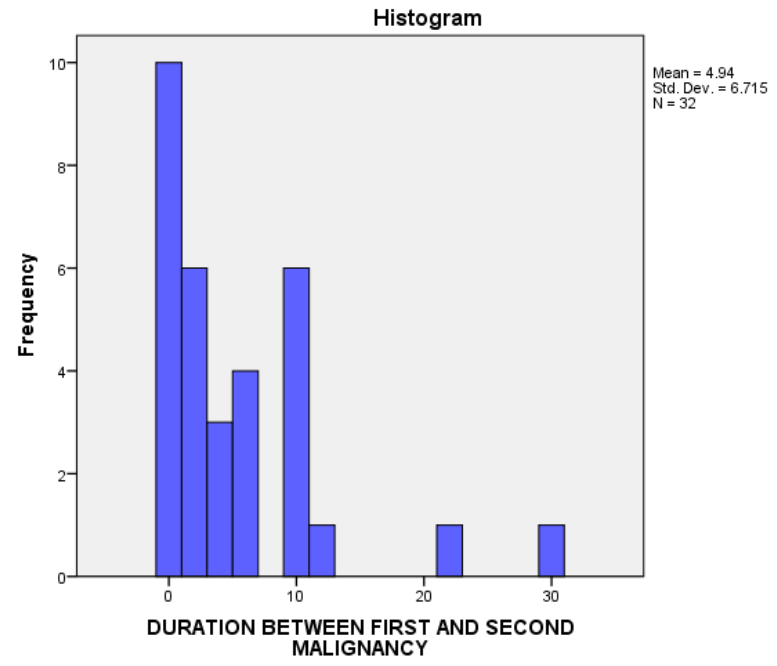

Figure 1: Duration between first and second primary malignancy

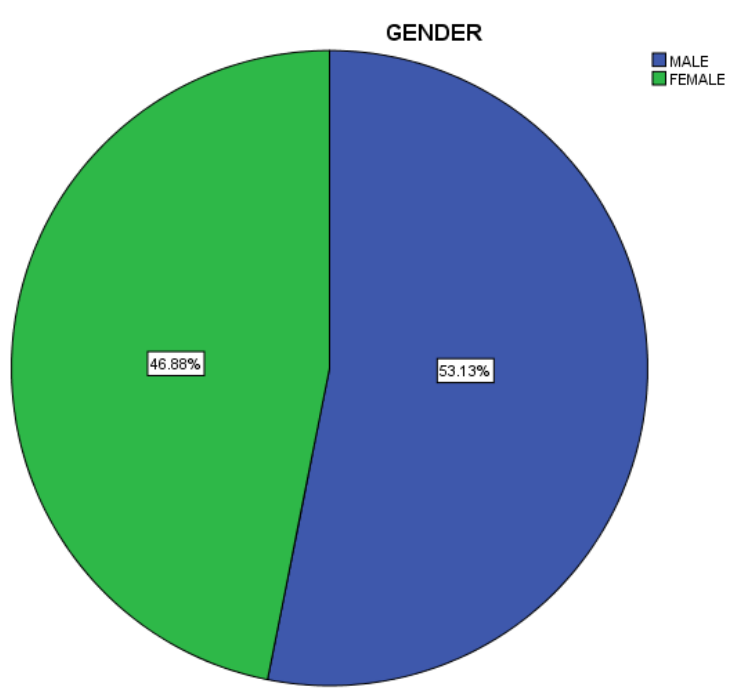

Figure 2: Gender Distribution

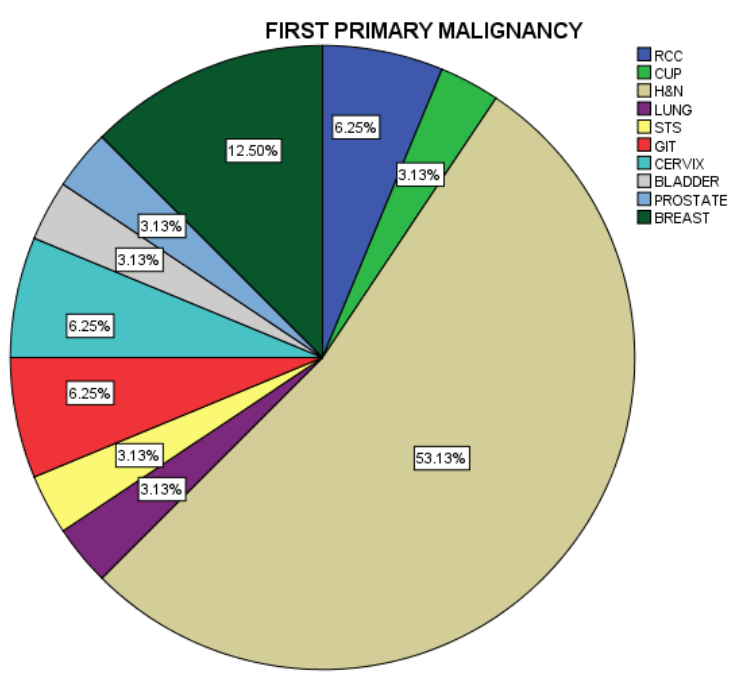

Figure 3: First Primary Malignancy

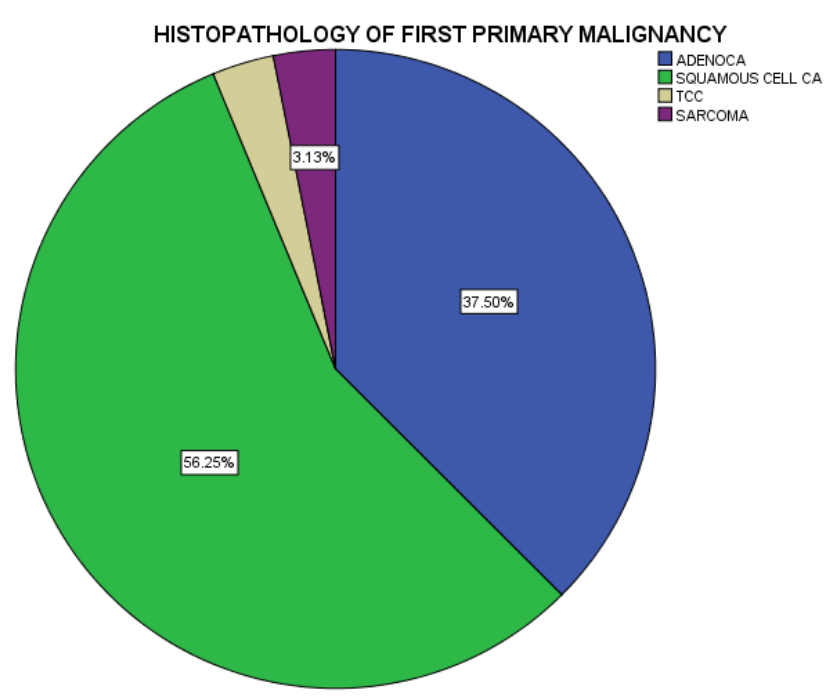

Figure 4: Histopathology of First Primary Malignancy

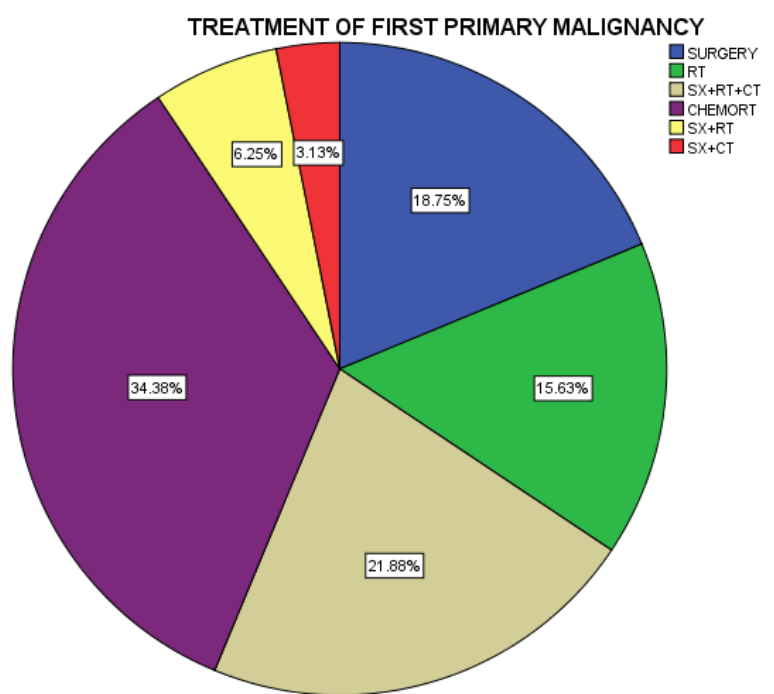

Figure 5: Treatment of First Primary Malignancy

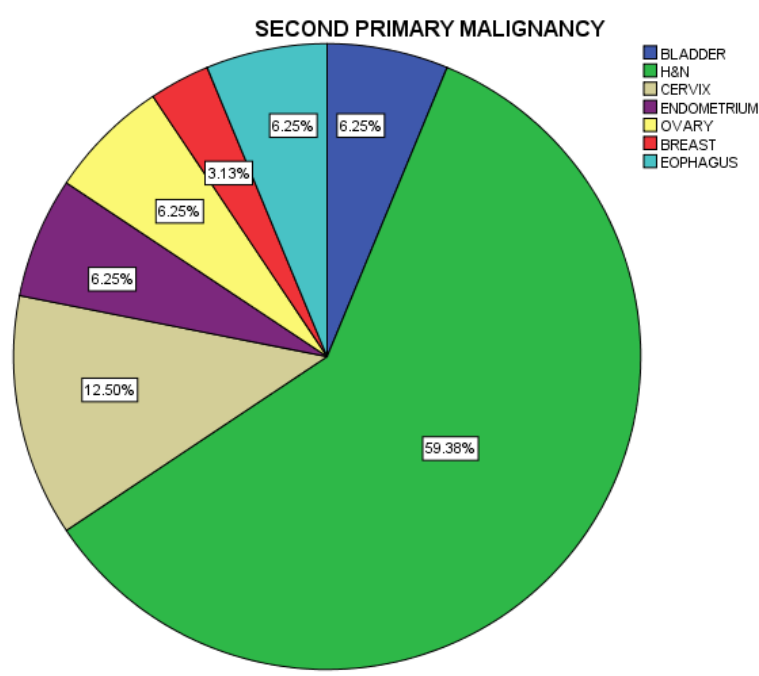

Figure 6: Second Primary Malignancy 


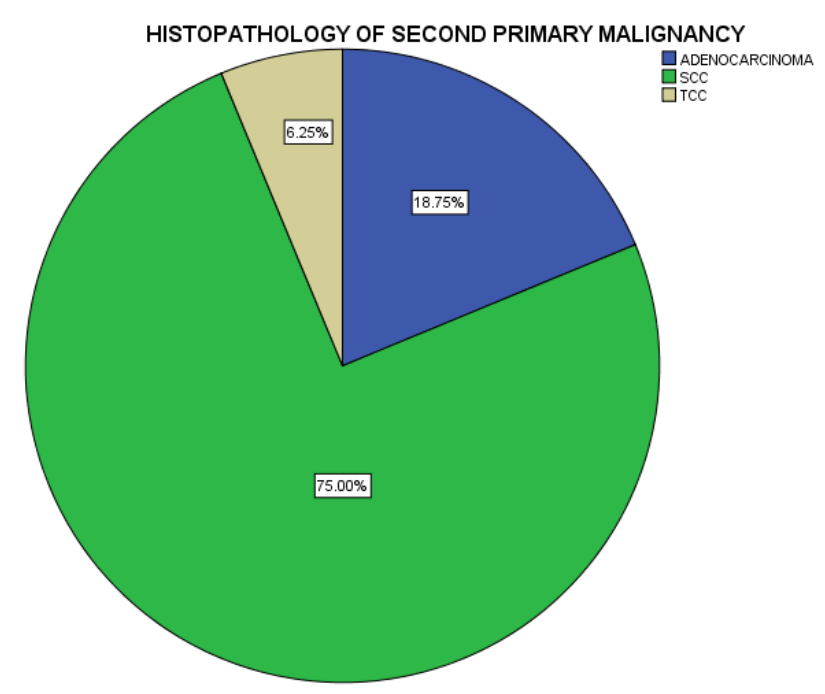

Figure 7: Histopathology of second primary malignancy

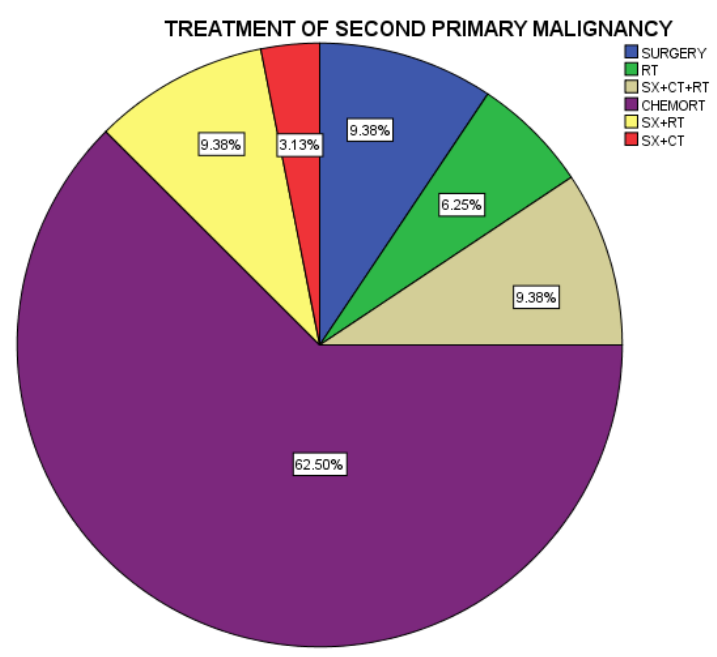

Figure 8: Treatment of Second Primary

\section{Discussion}

As the number of cancer survivors' increase with better therapeutic modalities for cancer, the number of patients with multiple primary malignancies will also increase. Patients with index cancer diagnosed at a younger age, patients with an indolent cancer with longer survival are at an increased risk of developing metachronous malignant neoplasm. Patients with a positive family history, exposure to common environmental carcinogens, patients with cancer predisposition syndromes are more susceptible for developing multiple cancers ${ }^{(8)}$.

The incidence of multiple primary malignant neoplasms in a patient with breast cancer is between $4.1 \%$ and $16.4 \%$. Second malignancy develops after a median time interval of 5 to 8 years $^{(8}$ to ${ }^{12)}$. Hormonal and genetic factors (BRCA1, BRCA2gene) are responsible for development of multiple primaries in patients with breast cancer $^{(12)}$. Breast cancer patients are more prone to develop endometrial, ovary, gastric and colon cancers. There was association between breast and cervix, breast and endometrium, breast and head and neck cancer in our study.

There is an increased risk of bladder and colorectal cancer in prostate cancer patients treated with external beam radiotherapy ${ }^{(13,14)}$. One prostate cancer patient in our study had developed synchronous male breast cancer. Hodgkins lymphoma survivors treated with radiotherapy are at an increased risk of developing breast, thyroid, lung and colorectal cancers. Patients treated with combination chemotherapy are more prone to develop secondary leukemias ${ }^{(15,16)}$. Lung cancer patients are more prone to develop second malignancies which are related to smoking ${ }^{(17)}$. There was an association between lung cancer and cervical cancer in our study.

Cancer predisposition syndromes must be ruled out in patients with multiple primary malignant neoplasms, patients with a strong family history and in patients who are diagnosed with cancer at a younger age. The most common cancer predisposition syndromes are Hereditary breast and ovarian cancer syndrome, Lynch syndrome, Von Hippel Lindau disease, multiple endocrine neoplasia and Li Fraumeni syndrome. Common clinical scenarios of patients with potential genetic syndrome are occurrence of bilateral breast cancer, breast and ovarian cancer, breast cancer and sarcoma, colon and endometrial cancers, colon and ovarian cancers, multiple renal cell carcinomas $^{(18)}$. We had 3 patients with potential genetic syndrome (breast and endometrium, breast and prostate cancer, sarcoma and endometrial cancer).

Treatment of patients with multiple primary malignant neoplasm warrants multidisciplinary management and the treatment needs to be adapted and individualised to face the therapeutic 
challenges. A systemic chemotherapy regimen which is effective for both the primary malignancies must be chosen. For patients requiring reirradiation, the tolerance of previously irradiated tissue should be taken into consideration.

\section{Conclusion}

The incidence of multiple primary malignant neoplasms is on the rise due to better diagnostic modalities and longer survival of cancer patients due to effective therapeutic strategies. A regular follow up, a strong clinical suspicion and thorough evaluation could detect most of the metachronous second malignancies at an earlier stage. An individualised treatment approach is warranted for patients with multiple malignancies. The prognosis of these patients depends on the individual tumor biology and the stage of presentation of the individual tumor.

\section{References}

1. Coyte A, Morrison DS, McLoone P. Second primary cancer risk - the impact of applying different definitions of multiple primaries: results from a retrospective population-based cancer registry study. BMC Cancer 2014;14:272.

2. Buiatti E, Crocetti E, Acciai S, et al. Incidence of second primary cancers in three Italian population-based cancer registries. Eur J Cancer 1997;33:1829-34.

3. Weir HK, Johnson CJ, Thompson TD. The effect of multiple primary rules on population-based cancer survival. Cancer Causes Control 2013;24:1231-42.

4. Rosso S, De Angelis R, Ciccolallo L, et al. Multiple tumours in survival estimates. Eur J Cancer 2009;45:1080-94.

5. Karaholios E, English D, Thursfield V, et al. Second primary cancers in Victoria. 2013. http://www. cancervic. org.

6. Moertel CG. Multiple primary malignant neoplasms: Historical perspectives. Cancer 1977;40(Suppl 4):1786-92.
7. Warren S, Gates O. Multiple primary malignant tumors: A survey of the literature and statistical study. Am J Cancer. 1932; 16:1358-414.

8. Amer MH. Multiple neoplasms, single primaries, and patient survival. Cancer Manag Res 2014;6:119-34.

9. Weir HK, Johnson CJ, Thompson TD. The effect of multiple primary rules on population-based cancer survival. Cancer Causes Control 2013;24:1231-42.

10. Kim JY, Song HS. Metachronous double primary cancer after treatment of breast cancer. Cancer Res Treat 2015;47:64-71.

11. Ricceri F, Fasanelli F, Giraudo MT, et al. Risk of second primary malignancies in women with breast cancer: results from the European prospective investigation into cancer and nutrition (EPIC). Int $J$ Cancer 2015;137:940-8.

12. Molina-Montes E, Pérez-Nevot B, Pollán $\mathrm{M}$, et al. Cumulative risk of second primary contralateral breast cancer in BRCA1/BRCA2 mutation carriers with a first breast cancer: a systematic review and meta-analysis. Breast 2014;23:721-42.

13. Jin T, Song T, Deng S, et al. Radiationinduced secondary malignancy in prostate cancer: a systematic review and metaanalysis. Urol Int 2014;93:279-88.

14. Wallis CJ, Mahar AL, Choo R, et al. Second malignancies after radiotherapy for prostate cancer: systematic review and meta-analysis. BMJ 2016;352:i851.

15. Bhuller KS, Zhang Y, Li D, et al. Late mortality, secondary malignancy and hospitalisation in teenage and young adult survivors of hodgkin lymphoma: report of the childhood/adolescent/young adult cancer survivors research program and the BC cancer agency centre for lymphoid cancer. Br J Haematol 2016;172:757-68.

16. Lisik-Habib M, Czernek U, DębskaSzmich S, et al. Secondary cancer in a survivor of hodgkin's lymphoma: a case 
report and review of the literature. Oncol Lett 2015;9:964-6.

17. Sanchez De Cos Escuin J, Rodriguez Lopez DP, Delgado Utrabo I, et al. Disease recurrence and second tumors in Long-term survivors of lung Cancer. Arch Bronconeumol 2016;52:183-8.

18. Garber JE, Offit K. Hereditary cancer predisposition syndromes. J Clin Oncol 2005;23:276-92. 